\title{
Coexistência Wi-Fi x LTE: uma análise comparativa de desempenho entre o LAA e o LTE-U
}

\author{
Daniel Luzente de Lima, Marcelo Gonçalves Rubinstein
}

Resumo-LTE é o acrônimo para Long Term Evolution, padrão de rede móvel da quarta geração (4G). O LTE trouxe novas técnicas desde algoritmos de gerenciamento de recursos ao aumento da largura de banda. Enquanto o $5 \mathrm{G}$ não é implantado, a demanda por recursos cresce, o que motiva estudos para novas implementações. O LTE opera em faixas licenciadas dentro do espectro, enquanto o Wi-Fi faz uso de faixas públicas. É nesse contexto que surge o tema da coexistência das redes Wi-Fi e LTE. As tecnologias para coexistência das redes mais conhecidas são Licensed-Assisted Access (LAA) e LTE-Unlicensed (LTE-U). Neste artigo serão apresentados resultados comparativos entre os padrões LAA e LTE-U, voltados aos impactos causados em usuários da rede Wi-Fi durante o compartilhamento da frequência $5 \mathrm{GHz}$. Os resultados mostram que o método de acesso justo no compartilhamento da frequência pública do espectro usado no LAA diminui em torno de $58 \%$ o impacto nos usuários da rede Wi-Fi.

Palavras-Chave-LTE, LAA, LTE-U, 4G, 5G, Wi-Fi.

Abstract-LTE stands for Long Term Evolution, the fourth generation (4G) mobile network standard. It brought new techniques, from resource management algorithms to increasing bandwidth. While 5G is not deployed, the demand for resources grows, which motivates studies for new implementations. LTE operates on licensed bands within the spectrum, while WiFi makes use of public bands. It is in this context that the theme of the coexistence of Wi-Fi and LTE networks arises. The technologies for network coexistence most well-known are Licensed-Assisted Access (LAA) and LTE-Unlicensed (LTE-U). In this paper, comparative results between the LAA and LTE-U standards will be presented, aimed at impacts caused on users of the Wi-Fi network during the sharing of the $5 \mathrm{GHz}$ frequency. Results show that the fair access method for sharing the public frequency of the spectrum used on LAA decreases around $58 \%$ the impact on users of the Wi-Fi network.

Keywords-LTE, LAA, LTE-U, 4G, 5G, Wi-Fi.

\section{INTRODUÇÃO}

As redes sem fio, que possuem uso massivo e diversificado de dispositivos como celulares, notebooks, além de diversos eletrônicos, evoluíram para acomodar toda essa demanda de tráfego e aplicações em diversos padrões desde o $\mathrm{Wi}-\mathrm{Fi}$, o Bluetooth em redes locais, até as redes de telefonia móvel com o $1 \mathrm{G}$, o $2 \mathrm{G}$, o $2.5 \mathrm{G}$, o $3 \mathrm{G}$ e por fim o LTE, o padrão de rede móvel da quarta geração (4G). Em constante evolução e adotado na maioria dos países, o $4 \mathrm{G}$ trouxe novas técnicas que envolvem o gerenciamento de recursos, modulações e uma maior da largura de banda de transmissão [1]. Tais recursos fomentaram o desenvolvimento e a utilização em larga escala

Daniel Luzente de Lima e Marcelo Gonçalves Rubinstein, Programa de Pós-Graduação em Engenharia Eletrônica, UERJ, Rio de Janeiro-RJ, e-mail: daniel.luzente@gmail.com. de aplicações multimídia e em tempo real, que provocaram uma demanda por serviços de dados muito superior aos de voz.

O cenário acima é corroborado por pesquisas internacionais de importantes empresas do mercado de tecnologia, como por exemplo a Cisco Systems, que publica anualmente o Cisco Annual Internet Report, com análise e previsão global da mudança de negócios voltados para telecomunicações, contemplando desde a banda larga fixa, o Wi-Fi até a telefonia móvel (3G, LTE, etc). Importantes projeções são mostradas ao lado de dados já consolidados do mercado em anos anteriores, indicando o crescimento de usuários da Internet, dispositivos e conexões, além de desempenho de rede e demandas de aplicativo.

A próxima geração da telefonia móvel, o $5 \mathrm{G}$, com previsão de início no Brasil em 2022, é o novo padrão com velocidades que deverão superar os $100 \mathrm{Mb} / \mathrm{s}$ de downlink e $50 \mathrm{Mb} / \mathrm{s}$ de uplink do seu antecessor, com uma latência muito menor, algo em torno $4 \mathrm{~ms}$, contra os $20 \mathrm{~ms}$ do padrão anterior. Isso favorecerá aplicações em tempo real, por exemplo. No entanto, não é o mais adequado considerar que o $5 \mathrm{G}$ substituirá $4 \mathrm{G}$, pois ambas as tecnologias terão um longo período de transição, operando em conjunto.

Enquanto o 5G não se torna uma realidade, a demanda por recursos de comunicação só aumenta, o que motiva iniciativas de pesquisas de novas implementações dentro do LTE, de forma a extrair do padrão melhor desempenho e certamente disponibilizar mais banda [1], principalmente pelo fato de que estudos indicam uma subutilização das faixas de frequência de 0 a $6 \mathrm{GHz}$, conforme pesquisa realizada pelo FCC (Federal Communications Commission) e ilustrada na Figura 1 [2] que mostra um vazio de detecção de energia justamente na faixa de $5 \mathrm{GHz}$, corroborando a ideia de que há potencial para exploração da faixa sem que isto implique em risco de disponibilidade para os dispositivos nativos, como por exemplo o Wi-Fi.

É nesse contexto que surge o tema da coexistência das redes Wi-Fi e LTE, ou Redes de Rádio Cognitivas. Segundo Akyildiz et al. [3], um Rádio Cognitivo é definido como um rádio que pode mudar seus parâmetros de transmissão baseandose na interação com o seu ambiente. Na prática, trata-se de uma rede sem fio inteligente que possui conhecimento do seu meio físico circundante e capacidade de aprender em temporeal com as variações estatísticas dos estímulos de entrada RF (Rádio Frequência), fazendo alterações correspondentes em certos parâmetros operacionais (por exemplo, potência de transmissão, frequência portadora e modulação) [4]. Em outra pesquisa relacionada ao tema [5], foi construído um 
framework específico para coexistência das redes LTE e Wi$\mathrm{Fi}$, com apresentação e testes comparativos dos padrões LAA e LTE-U, operando em faixas compartilhadas com rede WiFi. Assim como no artigo supramencionado, em [6] os autores também concluíram que o LAA é uma solução promissora para coexistência; no entanto os testes foram feitos com base em um cenário com baixa densidade (dois usuários) e apenas foi avaliado o padrão com LBT com WiFi. Já o presente trabalho avaliou resultados de simulações com diferentes densidades, além de comparação com o LTE-U que é padrão de coexistência com dutycycle. A nossa avaliação verificou que em certos cenários o LAA se mostra melhor vizinho aos usuários da rede Wi-Fi que o LTE-U, porém nossos testes em diferentes densidades de redes mostram que o desempenho do LAA não se mantém quando temos uma rede com 160 usuários, por exemplo. Em [9], é abordada a reutilização de faixas de espectro através

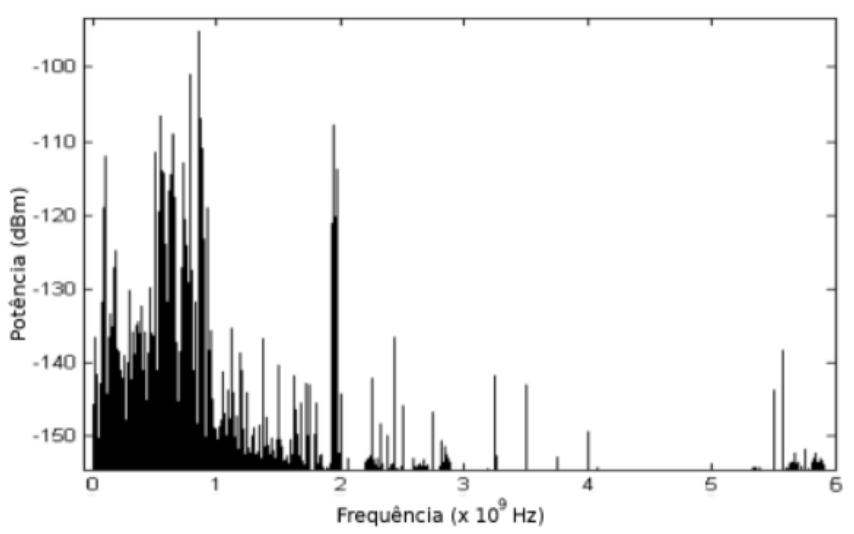

Figura 1: Utilização do espectro de 2 a $6 \mathrm{GHz}$. Imagem retirada de [7] [8].

de acesso dinâmico ao espectro (Dynamic Spectrum Access DSA). Já em [10] e [11] temos análises do LTE-U e LAA respectivamente, que são os padrões mais promissores para coexistência de redes em faixas frequências, corroborando que o aumento massivo do tráfego das redes sem fio vem motivando as pesquisas e o desenvolvimento de novas gerações de redes wireless.

O LTE opera em faixas licenciadas reguladas pela ANATEL e, por não sofrer com interferências de outras redes, já faz melhor uso possível da banda disponível. Contudo, há uma necessidade de aumento da banda em função da maior demanda. Uma solução é fazer uso de outras faixas para agregação de portadoras, no caso faixas não licenciadas, como por exemplo a de $2.4 \mathrm{GHz}$ ou $5 \mathrm{GHz}$. O problema é que essas faixas são públicas e usadas nas redes Bluetooth e WiFi. Logo, há necessidade de regulação rígida deste tipo de implementação, pois não se pode permitir que as operadoras esgotem as faixas de frequências públicas como a de $2.4 \mathrm{GHz}$ por exemplo, com tráfego dos seus clientes, inviabilizando o uso pelos dispositivos Bluetooth ou Wi-Fi.

Hoje temos uma série de implementações com o mesmo objetivo da coexistência das redes de rádio: LTE-U, LAA, LTE Advanced, MulteFire, e outras. No entanto, esta coexistência das redes LTE x Wi-Fi somente deve ocorrer sob método de acesso ao meio de transmissão que garanta "assegurar o compartilhamento justo do espectro não licenciado com outros sistemas legados, tais como o Wi-Fi" [12]. A base desta premissa é o LBT (Listen Before Talk), que é um recurso disponível no LAA e introduzido pelo 3GPP, em sua versão 13, com a função de permitir aos rádios transmissores escutar (Listen) o ambiente antes de iniciar a transmissão (Talk), o que garantiria que não houvesse deterioração do meio que já é ocupado pelos sistemas legados, em particular o Wi-Fi.

Este artigo apresenta uma análise de desempenho dos dois padrões mais promissores de acesso e compartilhamento de faixas de frequência públicas do espectro, o LTE-U e o LAA. Esses métodos utilizam mecanismos de acesso ao meio distintos, baseados em contenção (LAA) ou controle do domínio do tempo, que visam basicamente permitir escoamento de tráfego com aumento de vazão, sem que isto implique impactos nocivos aos usuários das redes nativas da faixa pública de frequência. O objetivo aqui é avaliar o desempenho do padrão LAA, em comparação com o LTE-U, em diferentes cenários de quantidades de usuários, tendo em vista que este será o padrão a ser adotado pelas operadoras e fabricantes de dispositivos, permitindo coexistência justa entre usuários LTE/LAA e usuários Wi-Fi.

O presente trabalho está organizado da seguinte forma. A Seção II apresenta os principais padrões derivados do LTE, enquanto a Seção III apresenta uma proposta de avaliação de desempenho visando comparar os dois padrões voltados para compartilhamento de frequência do espectro público, que são LTE-U e LAA. Na Seção IV são apresentados alguns resultados obtidos acompanhados de análise comparativa. Finalmente, a Seção V conclui o trabalho e propõe novas pesquisas.

\section{TECNOLOGIAS DE COMPARTILHAMENTO DE ESPECTRO}

A evolução do LTE trouxe diversas implementações voltadas ao compartilhamento de frequências, cada uma com suas características específicas e objetivos estabelecidos. Nas seções à seguir são apresentados detalhes das duas principais iniciativas para acesso às faixas não licenciadas como mecanismo de complementação da banda disponível do 3GPP (3rd Generation Partnership Project) que é uma organização tecnológica do ramo de telecomunicações cujo trabalho está voltado para padronizar a criação, o envio e a reprodução de arquivos multimídia em telefones celulares e outros aparelhos wireless.

\section{A. LTE-U}

O LTE-U foi apresentado como proposta de um novo padrão de comunicações em banda não-licenciada para ser usado em redes LTE pelo que ficou conhecido como LTEU Forum, criado pela Verizon, em associação com AlcatelLucent, Samsung, Qualcomm e Ericsson. Tecnicamente o LTE-U foi concebido para operar em modo de ciclos prédefinidos de duração de transmissão ou dutycycles. Na prática, o mecanismo ocupa o canal por um período de tempo, seguido de outro período de pausa ou silenciamento, que permite a 
outras tecnologias como IEEE 802.11 fazer uso oportunístico do recurso disponível. Uma metodologia dinâmica de definição do tempo do dutycycle, tomando como base medições de potência no meio de transmissão pode ser implementada, visando o aumento do desempenho.

\section{B. $L A A$}

A demanda por banda nas redes móveis envolve naturalmente a necessidade de novas faixas de frequências para transmissão. No entanto, as redes $4 \mathrm{G}$ teriam alcançado o limite físico diante da escassez de frequências dentro daquelas definidas atualmente pelas regulações internacionais para utilização das redes móveis. Diante das complexidades econômicas e regulatórias de se buscar utilização das frequências altas, as pesquisas indicam que a faixa de $5 \mathrm{GHz}$ é a que possui melhor potencial os fabricantes de infraestrutura de telecomunicações e chips integrados. Por esta razão muitos estudos para utilização compartilhada das faixas de espectro não licenciadas vêm sendo realizados. Uma iniciativa promissora chama-se Licensed-Assisted Access operation (LAA), que se constitui de uma implementação em que se agrega as redes em duas células, a primeira operando na faixa licenciada, com requisitos de QoS, e a segunda célula na faixa não licenciada, oportunisticamente para acelerar a taxa de dados. Introduzido inicialmente em 2016, pelo 3GPP Release 13, o LAA permite que um LTE Base Station (BS) transmita dados na faixa não licenciada de $5 \mathrm{GHz}$ para escoamento de tráfego de download, enquanto a faixa licenciada fica responsável pelo dados de uplink e tráfego de controle da rede, tudo isso fazendo uso de um framework voltado para agregação de portadoras [13]. Posteriormente, o Release 14 adicionou a possibilidade do tráfego de uplink também ser transmitido pela faixa não licenciada, em um padrão que ficou conhecido como Enhanced LAA (LTE-eLAA) [18]. Os principais requisitos do LAA são:

- o LAA não pode ocasionar impactos em redes Wi-Fi mais do que qualquer outra rede Wi-Fi incluída no mesmo ambiente;

- uso de LBT quando em uso nas faixas não licenciadas;

- uso de SCELL (small cell), que são nós de acesso de rádio celular de baixa potência com alcance de 10 metros a alguns quilômetros, operando na faixa não licenciada;

- limite de detecção de energia (Energy Detection Threshold - EDT) para verificação de meio ocioso.

O espectro de $5 \mathrm{GHz}$ está disponível globalmente, porém um dispositivo operando nesta faixa deve obedecer às normas regulatórias, que variam em cada região do mundo. Além dos requisitos de uso somente interno, potência máxima de saída, densidade espectral de potência na banda, a operação do LTE em espectro não licenciado deve implementar seleção de frequência dinâmica (DFS), controle de potência de transmissão (TPC) e Listen Before Talk (LBT), de forma que se evite as interferências em radares [14]. A ANATEL, em sua agenda regulatória do biênio 2017-2018, estabeleceu no item 51 o seguinte tema a ser deliberado: "Elaborar regulamentação que permita o uso da faixa de $5 \mathrm{GHz}$ por operadoras de forma compartilhada no espaço e no tempo com tecnologias WiFi" [15]. Esta pauta surgiu diante da necessidade de se obter mais banda diante dos novos conteúdos digitais disponíveis e da escassez de faixas disponíveis no espectro. Adicione-se a isto o fato de estarem surgindo diversas tecnologias que permitem o compartilhamento do uso de faixas entre serviços de telecomunicações que, em regra, necessitam de autorização de acesso a radiofrequências e serviços ou aplicações que são dispensadas desse tipo de autorização. Na prática a ANATEL decidiu não alterar a regulamentação vigente, apenas definiu que os equipamentos que farão uso da coexistência de frequências para agregação de portadoras devem obrigatoriamente fazer uso do recurso LBT (Listen Before Talk) e que sejam estabelecidos critérios de certificação dos mesmos.

Considerando o tema da coexistência de redes móveis de tecnologias distintas (ex. LTE e Wi-Fi), destacamos os padrões LTE-U e LAA como aqueles que buscam a utilização efetiva das frequências não licenciadas para aumento da vazão de dados e, por esta razão, identificamos estes dois padrões como os adequados para o estudo em questão. Na Tabela 1 são descritas as principais características destas tecnologias.

Tabela 1: Características do LTE-U e do LAA

\begin{tabular}{|c|c|c|}
\hline & LTE-U & LAA \\
\hline Padronização & LTE-U Forum & 3GPP \\
\hline Frequências & $\begin{array}{l}5150-5250 \mathrm{MHz} \\
5725-5850 \mathrm{MHz}\end{array}$ & $5150-5925 \mathrm{MHz}$ \\
\hline $\begin{array}{l}\text { Compartilhamento } \\
\text { de portadoras }\end{array}$ & $\begin{array}{c}\text { Até quatro } \\
\text { portadoras não } \\
\text { licenciadas }\end{array}$ & $\begin{array}{c}\text { Até duas } \\
\text { portadoras não } \\
\text { licenciadas }\end{array}$ \\
\hline $\begin{array}{l}\text { Integração LTE } \\
\text { licenciado }\end{array}$ & $\begin{array}{c}\text { Downlink } \\
\text { suplementar }\end{array}$ & $\begin{array}{c}\text { Downlink e } \\
\text { Uplink }\end{array}$ \\
\hline Versão 3GPP & $\begin{array}{c}\text { Baseado nas } \\
\text { versões } 10,11 \mathrm{e} \\
12\end{array}$ & $\begin{array}{l}\text { Baseado na } \\
\text { versão } 13\end{array}$ \\
\hline $\begin{array}{l}\text { Coexistência } \\
\text { com Wi-Fi }\end{array}$ & dutycycle & LBT \\
\hline Regiões & $\begin{array}{l}\text { EUA, Coréia do } \\
\text { Sul, China, Índia, } \\
\text { etc. }\end{array}$ & $\begin{array}{l}\text { Europa, Japão, } \\
\text { Brasil }\end{array}$ \\
\hline
\end{tabular}

\section{Proposta de AVAliaÇão DE DESEMPENho}

\section{A. Ambiente de simulação}

Nesta seção detalhamos a configuração física e lógica do ambiente de simulação utilizado. Serão avaliados os dois padrões mais promissores e mais utilizados em pesquisas correlatas que são o LTE-U e o LAA. O objetivo primordial da simulação é aferir o desempenho dos nós das redes que compartilham frequências não licenciadas do espectro e os impactos que os nós secundários (LTE-U e LAA) causam aos nós primários da rede (Wi-Fi).

As simulações foram realizadas com o software ns-3 [16] com extensões voltadas para a coexistência de redes na faixa de $5 \mathrm{GHz}$ [17]. 


\section{B. Topologia e parâmetros da rede}

$\mathrm{Na}$ Tabela 2, se encontram os parâmetros de configuração da rede utilizados nas simulações. Tabela 2: Parâmetros LTEU/LAA x Wi-Fi.

\begin{tabular}{|c|c|}
\hline \multicolumn{2}{|c|}{ Parâmetros Gerais } \\
\hline Qtde. de Estações Base & 4 \\
\hline Qtde. de hosts & 4,20 e 80 \\
\hline Distância entre os nós & Randômica \\
\hline Protocolo de Transporte & UDP \\
\hline Fontes e Destinos & Fonte fixa I Destinos aleatórios \\
\hline Tamanho Pacote & $512 \mathrm{~KB}$ \\
\hline Taxa de Transmissão & $150 \mathrm{Mbps}$ \\
\hline \multicolumn{2}{|c|}{ Parâmetros LAA } \\
\hline Acesso ao meio & Listen Before Talk (LBT) \\
\hline Detecção de Energia & $62 \mathrm{dBm}$ \\
\hline LAA - DRS & $80 \mathrm{~ms}$ \\
\hline LAA - TxOp & $8 \mathrm{~ms}$ \\
\hline \multicolumn{2}{|c|}{ Parâmetros LTE-U } \\
\hline Acesso ao meio & dutycycle \\
\hline Largura de banda & $20 \mathrm{MHz}$ \\
\hline ON/OFF duration & $20 \mathrm{~ms}$ \\
\hline
\end{tabular}

\section{RESULTADOS}

Os resultados das simulações estão apresentados nas figuras à seguir, onde temos a vazão comparativa das redes LAA e LTE-U e seus impactos nos usuários das redes de espectro aberto, que no caso deste trabalho é a rede Wi-Fi. Os histogramas azuis correspondem ao LAA e ao Wi-Fi sendo utilizados ao mesmo tempo e os vermelhos estão relacionados ao LTE$\mathrm{U}$ e ao Wi-Fi também transmitindo simultaneamente. A rede LAA utiliza o método LBT de acesso ao meio e o LTE-U o dutycycle, conforme padrões de suas respectivas definições técnicas. Foram executadas dez simulações para cenário com LAA e Wi-Fi e outras dez para LTE-U coexistindo com o Wi-Fi. Como mencionado anteriormente utilizamos a vazão como métrica nos gráficos apresentados à seguir, com médias e intervalos de confiança de 95\%. De acordo com a Figura 2, em

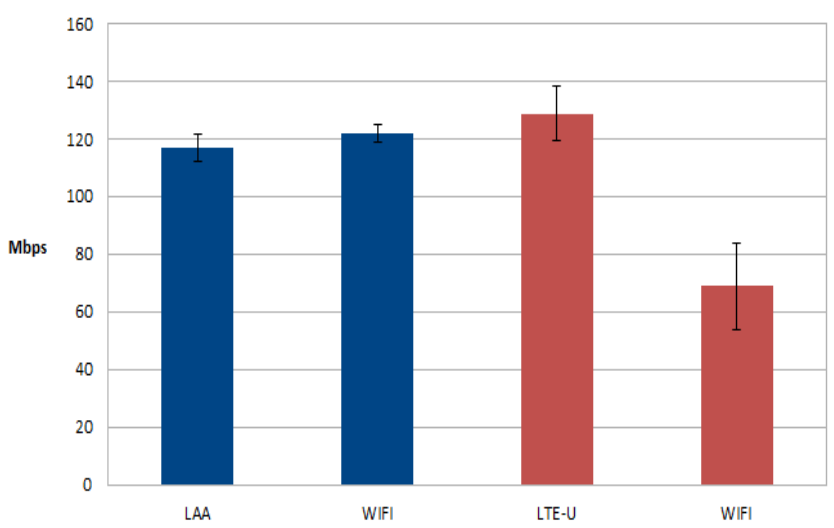

Figura 2: Vazão quando a densidade da rede é baixa - total de 8 usuários. uma rede menos densa (4 usuários LAA/LTE-U e 4 usuários Wi-Fi), verifica-se que os vizinhos que utilizam o padrão LAA e seu método de acesso baseado em contenção (LBT) são menos impactantes aos usuários da rede de espectro aberto que os usuários utilizando o mecanismo de acesso ao meio baseado por controle de tempo (dutycycle). Esse resultado era esperado tendo em vista que o LTE-U, ao transmitir na faixa não licenciada, não faz qualquer verificação sobre a ocupação do meio, apenas transmite alternando entre os estados ON e OFF. Isto faz com que os usuários da rede WiFi experimentem muitas colisões e retransmissões, impactando severamente a vazão média. Já no cenário de coexistência do Wi-Fi com o LAA, que realiza o processo de LBT antes de cada transmissão, os usuários da rede licenciada alcançam desempenho muito próximo do LTE-U, porém com menos impacto aos usuários nativos da faixa não licenciada. Os dispositivos LAA obtiveram vazão média de $115,50 \mathrm{Mbps}$, enquanto os dispositivos LTE-U alcançaram 129,14 Mbps, contudo, a vazão máxima dos usuários da rede Wi-Fi com vizinhos LAA transmitindo simultaneamente obtiveram em média 122,15 Mbps, enquanto que coexistindo com dispositivos LTE-U, os usuários do Wi-Fi transmitiram em média apenas 66,70 Mbps. Em resumo, em uma rede pouco densa, o impacto na rede Wi-Fi foi de $58 \%$, algo muito significativo se consideramos a pouca quantidade de usuários da rede.

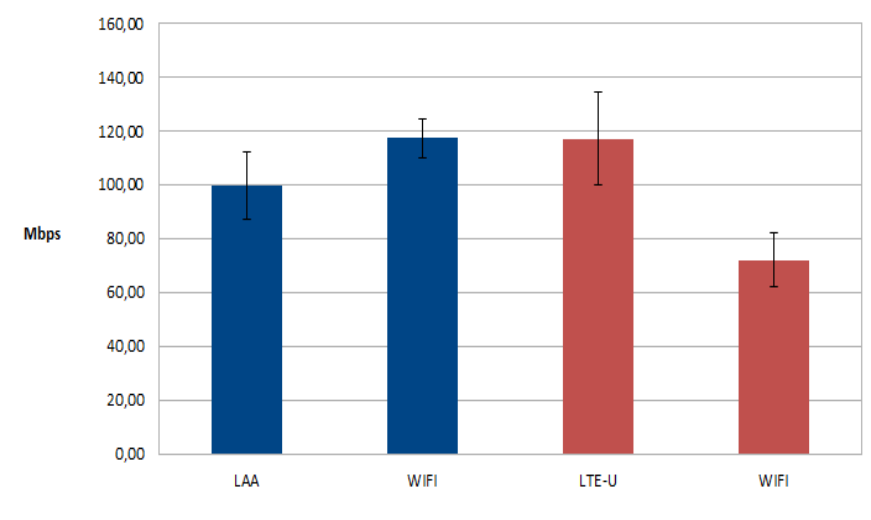

Figura 3: Vazão quando a densidade da rede é média - total de 40 usuários.

Conforme Figura 3, quando aumentamos a densidade da rede de 8 para 40 usuários, as vazões diminuem conforme esperado, com os dispositivos LAA e LTE-U obtendo 98,55 e 114,20 Mbps. Porém, assim como na rede pouco densa, o desempenho melhor do LTE-U contrasta com o desempenho dos vizinhos da rede de espectro aberto, Wi-Fi. O Wi-Fi com dispositivos não nativos que usam método LBT (LAA) para compartilhamento da faixa do espectro obtém 115,81 Mbps em média. Já com vizinhos que usam método dutycycle (LTEU) de acesso ao meio, o Wi-Fi alcança apenas 69,73 Mbps. Ou seja, o impacto negativo dos nós LTE-U nos usuários da rede Wi-Fi foi significativo, o que corrobora a análise anterior indicando que o método LBT de verificação de uso do meio de transmissão auxilia positivamente na coexistência das redes móveis LTE e Wi-Fi em uma mesma faixa do espectro. Em 


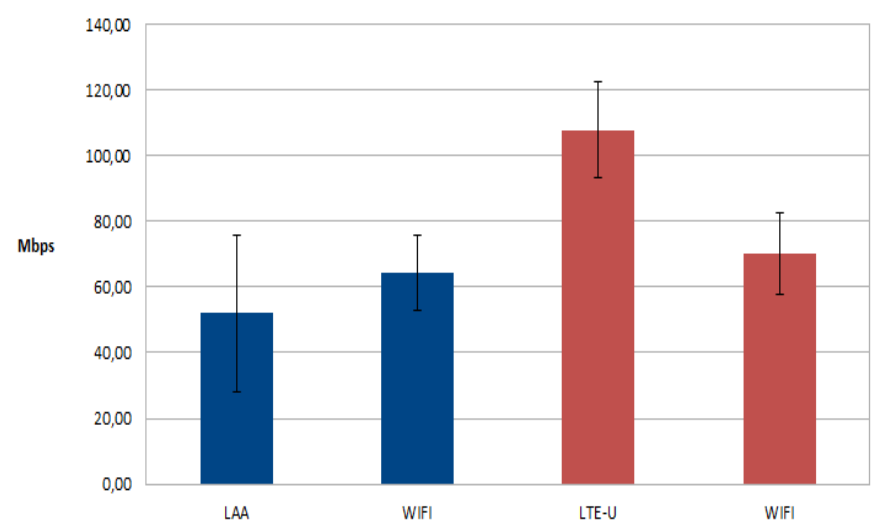

Figura 4: Vazão quando a densidade da rede é alta - total de 160 usuários.

um cenário com densidade super alta da rede, distribuindo 160 usuários na topografia, metade na rede nativa da faixa de espectro aberta (Wi-Fi) e a outra metade coexistindo e compartilhando com seus respectivos meios de acesso (LAA - LBT e LTE-U - dutycycle), verificamos uma mudança de comportamento considerável. Os resultados ilustrados na Figura 4 mostram que com o mecanismo de LBT tivemos significativo impacto nos dispositivos LAA, com vazão média de 54,47 Mbps em comparação com os 114,46 Mbps do LTEU. Já os usuários do Wi-Fi foram igualmente impactados por ambos os vizinhos, alcançando aproximadamente $66 \mathrm{Mbps}$ independente de qual tipo de dispositivo estava coexistindo e compartilhando o meio de transmissão. No entanto, a queda de desempenho das redes LAA e Wi-Fi quando compartilham a frequência neste cenário mais denso foi mais significativa do que quando coexistem o LTE-U e o Wi-Fi. Isso pode ser explicado pelo aumento do tráfego de controle que cresceu em virtude da quantidade de usuários realizando tentativas de acesso ao meio de transmissão.

\section{CONClusões}

Com relação à coexistência dos dispositivos de redes licenciadas e não licenciadas, verificamos que os padrões LAA e LTE-U, apesar dos diferentes mecanismos de acesso ao meio, permitem a coexistência, com destaques para o significativo impacto que o LTE-U ocasiona nos dispositivos Wi-Fi, bem como o baixo desempenho dos nós LAA quando em redes muito densas. Considerando o cenário no Brasil, em que a ANATEL decidiu que o compartilhamento das faixas públicas de frequência deve ser realizado mediante dispositivos de rádio que utilizem métodos de acesso ao meio com LBT, verificase que na maioria dos cenários o desempenho mediante compartilhamento da frequência de $5 \mathrm{GHz}$ será satisfatório, exceto quando tivermos uma alta densidade da rede. Neste caso a vazão cai drasticamente tanto na rede LAA/LTE quanto para os usuários nativos da rede Wi-Fi. No entanto, trata-se de cenário não muito comum podendo não ser um problema tão significativo.

Trata-se de um padrão relativamente novo, e que ainda pode ser explorado e otimizado visando melhor desempenho, porém sem dúvidas está pronto para uso e oferece excelente condição para acesso adequado e justo às faixas públicas de frequência do espectro.

Em trabalhos posteriores, considera-se importante realizar simulações com diferentes parâmetros como por exemplo DRS, cujo padrão do LAA é de $80 \mathrm{~ms}$, porém podendo ser configurado para 40 ou $160 \mathrm{~ms}$.

\section{AGRADECIMENTOS}

O presente trabalho foi realizado com apoio da Coordenação de Aperfeiçoamento de Pessoal de Nível Superior - Brasil (CAPES) - Código de Financiamento 001, da FAPERJ, do CNPq e da Diretoria de Infraestrutura Tecnológica do Centro de Tecnologia da Informação e da Comunicação do Estado do RJ (PRODERJ).

\section{REFERÊNCIAS}

[1] LAA and LTE-U Coexistence Simulator. Accessed: Jun. 4, 2019. [Online]. Available: http://bitbucket.org/cttc-lena/ns-3-lena-dev-lte-u.

[2] Cisco Annual Internet Report (2018-2023). https://www.cisco.com/c/en/us/solutions/collateral/executiveperspectives/annual-internet-report/white-paper-c11-741490.html.

[3] I. F. Akyildiz, W. Y. Lee, M. C. Vuran and S. Mohanty. "A survey on spectrum management in cognitive radio networks", IEEE Communications Magazine, vol. 46, no. 4, pp. 40-48, 2008.

[4] Rocha, U.; Olimpio, T.; Sobral, A. A. L.; Araujo, C. A.; Sousa, Marcelo Portela; Rocha, J. S. . Simulação com Redes de Rádios Cognitivos. VI Encontro Anual do Iecom em Comunicações, Redes e Criptografia (Encom 2016), 2016, Fortaleza - CE.

[5] B. Bojović, L. Giupponi, Z. Ali and M. Miozzo, Evaluating Unlicensed LTE Technologies: LAA vs LTE-U, in IEEE Access, vol. 7, pp. 8971489751, 2019

[6] V. A. d. S. J. Pedro Maia de Santana, Vinícius Dantas de Lima Melo, "Desempenho da Coexistencia LTE-LBT/Wi-Fi," XXXIV Simpósio Brasileiro de Telecomunicações - SBrT, 2016.

[7] Sousa, M. P. et al. Redes Cognitivas: Um Novo Paradigma para as Comunicações Sem Fio. Minicursos do XXVIII Simpósio Brasileiro de Redes de Computadores e Sistemas Distribuídos, p. 153-190, 2010.

[8] D. Cabric, S. M. Mishra and R. W. Brodersen, Implementation issues in spectrum sensing for cognitive radios, Conference Record of the ThirtyEighth Asilomar Conference on Signals, Systems and Computers, 2004, pp. $772-776$ Vol.1.

[9] I. F. Akyildiz, W. Lee, M. C. Vuran and S. Mohanty, "A survey on spectrum management in cognitive radio networks,"in IEEE Communications Magazine, vol. 46, no. 4, pp. 40-48, April 2008.

[10] Abdel-Rahman, Mohammad J., Abdelraheem, Mohamed, Mackenzie Allen, Cardoso, Kleber, Krunz, Marwan. On the orchestration of robust virtual LTE-U networks from hybrid half/full-duplex Wi-Fi APs. IEEE Wireless Communications and Networking Conference (WCNC), Doha., 2016. p. 1

[11] B. Chen, J. Chen, Y. Gao and J. Zhang, Coexistence of LTE-LAA and Wi-Fi on $5 \mathrm{GHz}$ With Corresponding Deployment Scenarios: A Survey, IEEE Communications Surveys Tutorials, vol. 19, no. 1, pp. 7-32, Firstquarter 2017.

[12] Guedes, R. M.; Silva, M. W. R.; Coutinho, P. S.; Rezende, J. F. . Redes de Rádios Cognitivos Utilizando Sequências de Saltos Baseadas em Papéis. Simpósio Brasileiro de Redes de Computadores e Sistemas Distribuídos, 2013, Brasília, p. 673-686.

[13] P. Kutsevol, V. Loginov, E. Khorov and A. Lyakhov, Analytical study of license-assisted access in $5 \mathrm{G}$ networks, IFIP Networking Conference, 2019, pp. 1-9.

[14] H. -J. Kwon et al., Licensed-Assisted Access to Unlicensed Spectrum in LTE Release 13, in IEEE Communications Magazine, vol. 55, no. 2 , pp. 201-207, February 2017.

[15] SEI/ANATEL - 3349698 - Análise (Processo n ${ }^{\circ} 53500.035604 / 2018$ 30)

[16] https://www.nsnam.org/wiki/LAA-WiFi-Coexistence

[17] LAA and LTE-U Coexistence Simulator. Disponível em: http://bitbucket.org/cttc-lena/ns-3-lena-dev-lte-u

[18] J. Zhang, M. Wang, M. Hua, T. Xia, W. Yang, and X. You, "Lte on license-exempt spectrum," IEEE Communications Surveys e Tutorials, vol. 20,no. 1, pp. 647-673, 2017. 